\title{
Triiodothyronine alleviates alcoholic liver disease injury through the negative regulation of the NLRP3 signaling pathway
}

\author{
XIANG DONG $^{1 *}$, HONGMEI YANG $^{2 *}, \mathrm{CONG} \mathrm{LI}^{3}$, QI LIU ${ }^{4}$, QINGLIN BAI ${ }^{5}$ and ZHAORAN ZHANG ${ }^{1}$ \\ ${ }^{1}$ Department of Gastroenterology, Jining First People's Hospital, Jining, Shandong 272000; \\ ${ }^{2}$ Laboratory Medicine, Yantaishan Hospital, Yantai, Shandong 264000; Departments of ${ }^{3}$ Otolaryngology, \\ ${ }^{4}$ Endocrinology and ${ }^{5}$ Operation Room, People's Hospital of Zhangqiu District, Jinan, Shandong 250200, P.R. China
}

Received November 23, 2017; Accepted May 2, 2018

DOI: $10.3892 / \mathrm{etm} .2018 .6409$

\begin{abstract}
The aim of the present study was to investigate the effect and mechanism of triiodothyronine (T3) on alcoholic liver disease (ALD)-induced injuries in mice. A total of 40 male C57/BL6 mice were randomly divided into the Control, ALD, ALD+T3 and ALD+T3+AMP-activated protein kinase inhibitor (CC) groups. Mice were administered alcohol $(4 \mathrm{~g} / \mathrm{kg} / \mathrm{day})$ intragastrically for 4 weeks except for Control group. Mice in the ALD+T3 group were given $\mathrm{T} 3(0.1 \mathrm{mg} / \mathrm{kg} /$ day $)$ while mice in ALD+T3+CC group were given T3 $(0.1 \mathrm{mg} / \mathrm{kg} /$ day $)$ and CC $(10 \mathrm{mg} / \mathrm{kg} /$ day $)$ for 1 week. Control and ALD groups were treated with saline. Liver tissue and blood samples were obtained for testing. Alanine aminotransferase (ALT), aspartate aminotransferase (AST) and total bilirubin (TBIL) levels were determined and morphological changes in the liver tissues were observed under the optical microscope. Inflammatory factors, including IL-1 $\beta$ and transforming growth factor (TGF)- $\beta / 1, \alpha$-smooth muscle actin (SMA) and protein levels of nucleotide-binding oligomerization domain, leucine rich repeat containing family, pyrin domain containing 3 (NLRP3), caspase-1 and pro-IL-1 $\beta$ were measured. Serum ALT, AST and TBIL levels in the ALD+T3 group were significantly reduced compared with the ALD group, while they were significantly increased in the ALD+T3+CC group $(\mathrm{P}<0.05)$. The number of hepatic lobules in the ALD+T3 group was significantly reduced compared with the ALD group, whereas the number in the ALD+T3+CC group was significantly increased $(\mathrm{P}<0.05)$. IL- $1 \beta$ and TGF- $\beta 1$ levels in the ALD+T3 group were significantly decreased
\end{abstract}

Correspondence to: Dr Zhaoran Zhang, Department of Gastroenterology, Jining First People's Hospital, Building 3, 6 Jiankang Road, Jining, Shandong 272000, P.R. China

E-mail: zhanyulieyong@163.com

*Contributed equally

Key words: triiodothyronine, alcoholic cirrhosis, nucleotide-binding oligomerization domain, leucine rich repeat containing family, pyrin domain containing 3 compared with the ALD group; however, levels in the ALD+T3+CC group were significantly increased compared with the ALD+T3 group $(\mathrm{P}<0.05)$. In addition, it was revealed that the expression of $\alpha$-SMA mRNA and protein in the ALD+T3 group was significantly decreased compared with the ALD group, whereas it was significantly increased in the ALD+T3+CC group compared with the ALD+T3 group. Expression of NLRP3, caspase-1, IL-1 $\beta$ and TGF- $\beta 1$ in the ALD+T3 group was significantly decreased compared with the ALD group, while expression was significantly increased in the ALD+T3+CC group. Conversely, compared with the ALD group, expression of pro-IL-1 $\beta$ was significantly increased in the $\mathrm{ALD}+\mathrm{T} 3$ group and decreased in the $\mathrm{ALD}+\mathrm{T} 3+\mathrm{CC}$ group. In conclusion, T3 may reduce the inflammatory response and severity of liver cirrhosis in mice with ALD by negatively regulating the NLRP3 signaling pathway.

\section{Introduction}

Alcoholic liver disease (ALD), characterized by liver metabolic disorder, is an acute, chronic liver injury caused by long-term heavy drinking (1). The incidence of ALD has steadily increased in the last 10 years and it is now the second major cause of liver damage, secondary only to viral hepatitis (2). Acetaldehyde, the toxic product of alcohol metabolism, is the primary cause of ALD (3). According to previous studies, the interleukin-1 $\beta$ (IL-1 $\beta$ ) signaling pathway serves a role in lipid hepatitis, oxidative stress injury and apoptosis caused by chronic alcohol intake (4); its role is correlated with activation of the nucleotide-binding oligomerization domain, leucine rich repeat containing family, pyrin domain containing 3 (NLRP3) inflammasome (5).

NLRP3 has previously been reported as a key mediator of pro-inflammatory cytokine release (6). A number of studies have demonstrated that NLRP3 may also activate hepatic stellate cells and increase the severity of liver fibrosis; these mechanisms may be associated with the upregulation of transforming growth factor- $\beta 1$ (TGF- $\beta 1$ ) expression (7). Previous reports have confirmed that NLRP3 upregulates the expression of caspase-1, which mediates the transformation of pro-IL-1 $\beta$ into IL-1 $\beta(8,9)$. It has also been reported that triiodothyronine (T3) alleviates oxidative stress, inflammation and apoptosis caused by hepatic ischemia-reperfusion injury, and its mechanism of action is 
associated with negative regulation of the NLRP3 signaling pathway (10). Furthermore, Lycium barbarum has been reported to inhibit the NLRP3 inflammasome signaling pathway, thereby reducing alcohol-induced liver cell damage (11). Previous studies have confirmed that changes in thyroxine levels are associated with the severity of liver damage $(10,12)$. Serum and free T3 concentrations gradually decrease as the severity of liver disease increases, while their concentrations gradually increase as liver function improves following treatment (10). Serum thyroxine levels are generally low in patients with non-alcoholic hepatitis and are improved following the application of thyroxine receptor agonists (1). However, the mechanism of action remains unclear. It was revealed that liver function was significantly improved in patients with alcoholic cirrhosis following T3 treatment $(2,12)$. To study the underlying mechanism, the following animal experimental study was performed.

\section{Materials and methods}

Patients and treatments. A total of 10 patients (male: female, 7:3; mean age, $50 \pm 4.5$ years) with liver cirrhosis admitted to Jining First People's Hospital (Jining, China) were randomly selected from March to September 2016. The inclusion criteria were as follows: i) Compliance with the diagnostic criteria of alcoholic cirrhosis (13); ii) good medication compliance; iii) no serious complications. Exclusion criteria: i) liver cirrhosis caused by hepatitis B virus (HBV) or lithiase; ii) patients with contraindication of T3; iii) patients with thyroid diseases that affect this experiment. Blood samples were collected from median vein of elbow prior the treatment and alanine transaminase (ALT) and aspartate transaminase (AST) levels was measured. Patients were treated with T3 $(0.1 \mathrm{mg} / \mathrm{kg} / \mathrm{day}$, orally before eating) for 2 weeks. Further blood samples were collected and the ALT/AST content was measured again.

Animals and treatments. A total of 40 male C57/BL6 (age, 8 weeks; weight, 20-25 g) mice were purchased from Beijing Vital River Laboratory Animal Technology Co., Ltd. (Beijing, China), housed in a temperature controlled room $\left(21 \pm 2^{\circ} \mathrm{C}\right)$, relative humidity $40-60 \%$, on a $12 \mathrm{~h}$ light/dark cycle and provided with standard mice chow and water. Mice had free access to water and food. Mice were randomly divided into four groups: Control ( $n=10), \operatorname{ALD}(n=10), \operatorname{ALD}+\mathrm{T} 3(\mathrm{n}=10)$ and $\mathrm{ALD}+\mathrm{T} 3+\mathrm{AMP}$-activated protein kinase inhibitor $(\mathrm{CC} ; \mathrm{n}=10)$. $\mathrm{CC}$ is a commonly used inhibitor of the AMP-associated pathway (9). With the exception of the control group, all mice were treated with alcohol by gavage ( $4 \mathrm{~g} / \mathrm{kg} /$ day) for 4 weeks. Mice in the control group were treated with saline of the same volume. Afterwards, the ALD+T3 group was also treated with T3 by gavage $(0.1 \mathrm{mg} / \mathrm{kg} /$ day $)$ for 1 week, while the ALD+T3+CC group was treated with T3 $(0.1 \mathrm{mg} / \mathrm{kg} / \mathrm{day})$ and CC (10 mg/kg/day) by gavage for 1 week. Mice in the control and ALD groups were administered with the same amount of normal saline by gavage for 1 week. Following the successful establishment of the ALD model, mice were anesthetized with $10 \%$ chloral hydrate $(0.01 \mathrm{ml} / \mathrm{g})$ and euthanized by asphyxiation. In brief, anesthetized mice were placed in a sealed chamber and a $\mathrm{CO}_{2}$ input device was connected. $\mathrm{CO}_{2}$ was continuously injected at a flow rate of $30 \%$ volume per minute until the $\mathrm{CO}_{2}$ concentration in the chamber reached
$100 \%$. Following sacrifice, blood samples were collected from the tail and centrifuged at 2,500 x g, $4^{\circ} \mathrm{C}$ for $10 \mathrm{~min}$ to separate the serum, which was stored at $-80^{\circ} \mathrm{C}$ for subsequent analysis. Liver tissues were harvested via thoracotomy and the left lobes were fixed in $4 \%$ formaldehyde solution for standby application at room temperature for $24 \mathrm{~h}$. The remaining liver tissues were cut into small pieces $(2 \times 2 \mathrm{~mm})$ and stored at $-80^{\circ} \mathrm{C}$ prior to subsequent analysis. The present study was approved by the Medical Ethics Committee of Jining First People's Hospital and was performed in accordance with the regulations and procedures regarding human and animal subject protection. Patients or their guardians have provided written informed consent for publication.

Detection of serum ALT, AST and total bilirubin (TBIL). Serum transaminases (including ALT and AST) and TBIL levels may be used as indices for measuring liver damage (14). Serum samples from mice in each experimental group were analyzed using a fully automatic biochemical analyzer (Mindray, Shenzhen, China). The serum samples were diluted (1:10) using normal saline to the same ratio to detect the ALT, AST and TBIL content and the results were analyzed.

Detection of $I L-1 \beta, T G F-\beta 1$ and $\alpha$-smooth muscle actin $(\alpha-S M A)$ in liver tissues. Levels of IL-1 $\beta$, TGF- $\beta 1$ and $\alpha$-SMA were detected in liver tissues from mice in each group using the classical immunohistochemical method (MLB00C, MB100B, MAB1420; R\&D Systems, Inc., Minneapolis, MN, USA) as previously described (15). The results were statistically analyzed using SPSS version 18.0 (SPSS, Inc., Chicago, IL, USA).

Pathological changes in liver tissues. Liver tissues were fixed with $10 \%$ formaldehyde solution at room temperature for $24 \mathrm{~h}$, embedded in paraffin and sectioned into $5 \mu \mathrm{m}$ thickness according to previous methods (16), followed by hematoxylin and eosin staining as previously described (16). Pathological changes in liver tissues were observed under an optical microscope (magnification, $\mathrm{x} 400$ ) and the number of hepatic pseudo-lobules in each visual field selected a random was counted and compared by eye, followed by relevant statistical analysis using SPSS version 18.0 (SPSS, Inc.).

Reverse transcription-quantitative polymerase chain reaction $(R T-q P C R)$. Frozen liver tissue samples from mice in each group were thawed at room temperature and lysed using TRIzol (Invitrogen; Thermo Fisher Scientific, Inc., Waltham, MA, USA) for RNA detection. RNA was extracted using the RNeasy Plus Mini kit (Qiagen, Inc., Valencia, CA, USA). Relevant primer sequences of IL-1 $\beta$ (9), TGF- $\beta 1$ and $\alpha$-SMA (17) were as used in previous studies (Table I). Extracted RNA was reverse transcribed into cDNA by RT kit (K1622, Thermo Fisher Scientific, Inc., Waltham, MA, USA) and amplified by PCR using the following protocol: $95^{\circ} \mathrm{C}$ for $30 \mathrm{sec}$, followed by 40 cycles of $95^{\circ} \mathrm{C}$ for $5 \mathrm{sec}$ and $60^{\circ} \mathrm{C}$ for $31 \mathrm{sec}$. mRNA expression in each group was calculated using the $2^{-\Delta \Delta \mathrm{Cq}}$ method (18).

Western blotting. Frozen liver tissue samples from mice in each experimental group were thawed and protein was 
Table I. Primer sequences for reverse transcription-quantitative polymerase chain reaction.

\begin{tabular}{lll}
\hline Gene & Direction & \multicolumn{1}{c}{ Sequence (5'-3') } \\
\hline Interleukin-1 $\beta$ & Forward & AAAGCTCTCCACCTCAATTGG \\
& Reverse & TCGTTGCTTGTCTCTCCTTA \\
Transforming growth factor- $\beta 1$ & Forward & GCGGACTACTATGCTAAAGAGG \\
& Reverse & GTAGAGTTCCACATGTTGCTCC \\
$\alpha$-smooth muscle actin & Forward & TGACCCAGATTATGTTGAGACC \\
& Reverse & CCAGAGTCCAGCACAATACCA \\
GAPDH & Forward & GCGGGCGCTGGAGGAGAA \\
& Reverse & GGATCTTCATGAGGTAGTCAGTC \\
\hline
\end{tabular}

extracted using a radioimmunoprecipitation lysis buffer [PBS, $0.5 \%$ NP-40, $0.5 \%$ sodium deoxycholate, $0.1 \%$ sodium dodecyl sulfate, $5.5 \% \beta$-glycerophosphate, $1 \mathrm{mM}$ dithiothreitol and complete protease and phosphatase inhibitors (Roche Diagnostics, Basel, Switzerland)]. BCA was used to quantify the protein content. Protein lysate $(50 \mu \mathrm{g})$ was separated using 10\% SDS-PAGE gels. Following, proteins were transferred to polyvinylidene difluoride membranes. Membranes were blocked with 5\% milk at room temperature for $2 \mathrm{~h}$. Membranes were incubated overnight at $4^{\circ} \mathrm{C}$ with primary antibodies: Pro-IL-1 $\beta$ (1:500; ab14374; Abcam, Cambridge, MA, USA), IL-1 $\beta$ (1:500; ab150777; Abcam), TGF- $\beta 1$ (1:500; ab64715; Abcam), $\alpha$-SMA (1:500; ab124964; Abcam), NLRP3 (1:500; ab214185; Abcam), caspase-1 (1:500; ab1872; Abcam) and GAPDH (1:500; ab37168; Abcam). Followed by $1 \mathrm{~h}$ incubation at room temperature with goat-anti rabbit (1:500; sc2004; Santa Cruz Biotechnology, Inc., Dallas, TX, USA) or goat anti-mouse (1:500; sc2005; Santa Cruz Biotechnology, Inc.) horseradish peroxidase-conjugated secondary antibodies. Bands were quantified by densitometry using a Biosens SC-750 Gel Documentation system (Shanghai Bio-Tech Co., Ltd., Shanghai, China) and the relative expression of pro-IL-1 $\beta$, IL-1 $\beta$, TGF- $\beta 1, \alpha$-SMA, NLRP3 and caspase-1 were determined. GAPDH was used as the internal reference.

Statistical analysis. SPSS software version 18.0 (SPSS, Inc.) was used to analyze data, and all data are presented as the mean \pm standard deviation. The difference between two experimental groups was analyzed using a t-test and multiple group comparisons were performed using one-way analysis of variance followed by a least significant difference post hoc test. $\mathrm{P}<0.05$ was considered to indicate a statistically significant difference. Each experiment was repeated for three times.

\section{Results}

T3 improves liver function and reduces serum transaminase (ALT/AST) content in patients with ALD. It was revealed that patients' ALT/AST content following T3 application was significantly decreased compared with prior to the oral administration of T3 ( $\mathrm{P}<0.05$; Fig. 1).

T3 improves liver function and ameliorates liver damage in mice with alcoholic cirrhosis. The results demonstrated that

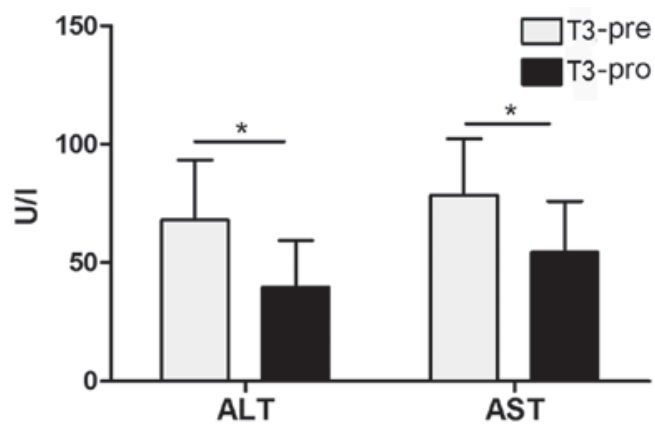

Figure 1. Comparison of serum ALT and AST in patients with alcoholic cirrhosis pre and pro T3. Data are presented as the mean \pm standard deviation. ${ }^{*} \mathrm{P}<0.05$. ALT, alanine transaminase; AST, aspartate transaminase; pre, prior to application; pro, post application; T3, triiodothyronine.

serum ALT/AST (Fig. 2A) and TBIL (Fig. 2B) levels in the ALD+T3 group were significantly reduced compared with those in the ALD group $(\mathrm{P}<0.05)$, whereas ALT/AST levels in the $A L D+T 3+C C$ group were significantly increased compared with those in the ALD+T3 group $(\mathrm{P}<0.05)$. These results suggest that $\mathrm{T} 3$ may effectively reduce the levels of liver enzymes and bilirubin in mice with alcoholic cirrhosis and have a significant protective effect on the liver.

Morphological analysis was performed on liver tissues collected from mice in each experimental group. It is accepted that pseudo-lobules are a pathological feature of liver cirrhosis (19). A number of hepatic lobules were observed in the experimental groups (Fig. 2C). The number of pseudo-lobules observed in the ALD+T3 group was significantly reduced compared with the ALD group $(\mathrm{P}<0.05)$, while the number of pseudo-lobules observed in the ALD+T3+CC group was significantly increased compared with the ALD+T3 group $(\mathrm{P}<0.01)$. These results suggest that $\mathrm{T} 3$ may alleviate the severity of liver fibrosis in mice with alcoholic cirrhosis. Taken together, these findings suggest that triiodothyronine improves liver function and alleviates the severity of alcoholic liver cirrhosis in mice, while its effect may be blocked by CC.

T3 downregulates IL-1 $\beta$ and TGF- $\beta 1$ in liver tissues. IL-1 $\beta$ (4) and TGF- $\beta 1$ (20) are important inflammatory mediators associated with the occurrence and development of alcoholic cirrhosis. Western blotting (Fig. 3A) and ELISA analysis revealed that the expression of IL-1 $\beta$ (Fig. 3B) and TGF- $\beta 1$ 
A

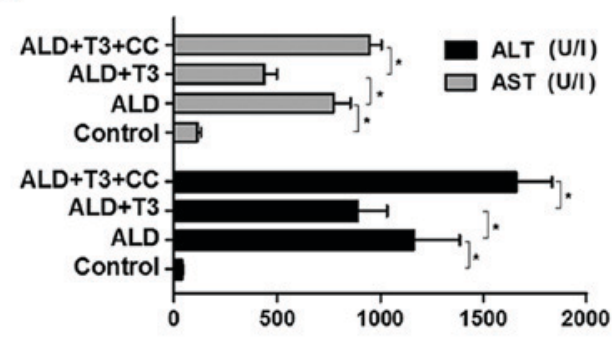

B

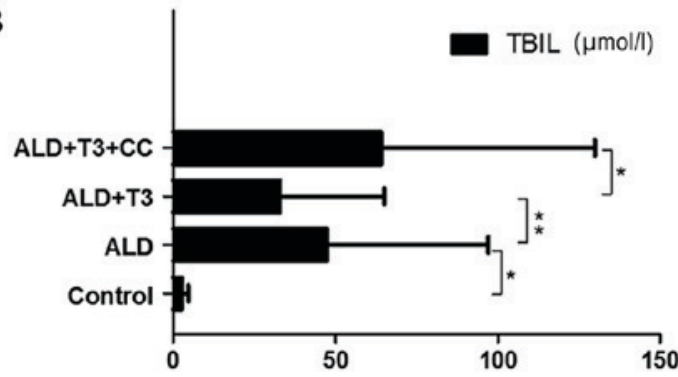

C

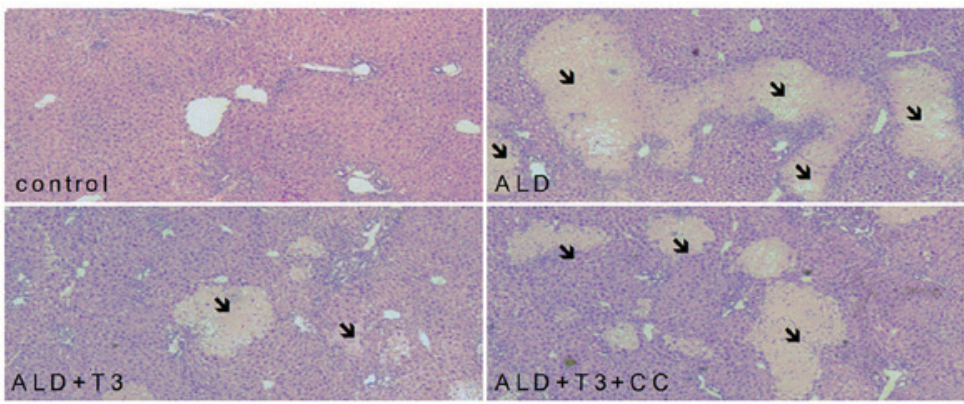

D

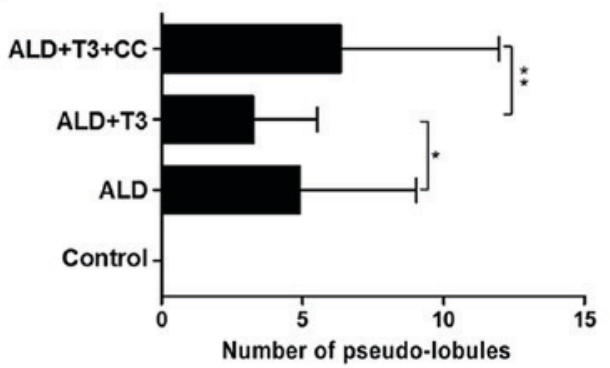

Figure 2. Comparison of serum (A) ALT, AST and (B) TBIL levels in each group (n=10). (C) Liver tissue samples were observed under an optical microscope (magnification, $\mathrm{x} 400$ ) following hematoxylin and eosin staining and (D) the number of pseudo-lobules in each group was counted and compared (n=10 visual fields). Data are expressed as the mean \pm standard deviation; black arrows indicate pseudo lobules. ${ }^{*} \mathrm{P}<0.05$ and ${ }^{* *} \mathrm{P}<0.01$. ALT, alanine transaminase; AST, aspartate transaminase; TBIL, total bilirubin; ALD, alcoholic liver disease; T3, triiodothyronine; CC, AMP-activated protein kinase inhibitor.

(Fig. 3C) mRNA and protein was significantly decreased in the ALD+T3 group compared with the ALD group $(\mathrm{P}<0.05)$. However, expression in the ALD+T3+CC group was significantly increased compared with the ALD+T3 group $(\mathrm{P}<0.05)$. These results suggest that $\mathrm{T} 3$ may effectively ameliorate the inflammatory response in mice with alcoholic cirrhosis and that this effect may be countered by $\mathrm{CC}$.

T3 reduces the expression of $\alpha$-SMA in liver tissues. Previous studies have demonstrated that the occurrence and development of liver cirrhosis is associated with the activation of hepatic stellate cells (21), which results in colloid and deposition. A key marker of hepatic stellate cell activation is the production of $\alpha$-SMA (22). In the present study, western blotting revealed that the expression of $\alpha$-SMA protein in the ALD+T3 group was significantly decreased compared with the ALD group $(\mathrm{P}<0.05)$; however, it was significantly increased in the ALD+T3+CC group compared with the ALD+T3 group $(\mathrm{P}<0.05$; Fig. 4). These results suggest that T3 may effectively reduce the $\alpha$-SMA content in the liver tissues of mice with alcoholic cirrhosis. It was also revealed that the level of
$\alpha$-SMA mRNA was significantly decreased in the ALD+T3 group compared with the ALD group $(\mathrm{P}<0.01)$, whereas it was significantly increased in the ALD+T3+CC group compared with the ALD+T3 group $(\mathrm{P}<0.05)$. These results reveal that $\mathrm{T} 3$ significantly reduces the expression of $\alpha$-SMA mRNA and protein in ALD mice and alleviates the severity of liver cirrhosis.

T3 negatively regulates the NLRP3-Caspase-1-pro-IL-1 $\beta$ $I L-1 \beta$ signaling pathway and the NLRP3-TGF- $\beta 1$ signaling pathway. To further study the molecular mechanism by which T3 alleviates liver damage in ALD mice, various associated proteins were detected. Western blotting (Fig. 5A) revealed that the expression of NLRP3, Caspase-1, IL- $1 \beta$ and TGF- $\beta 1$ in the ALD+T3 group was significantly decreased compared with the ALD group $(\mathrm{P}<0.05)$, whereas it was significantly increased in the $\mathrm{ALD}+\mathrm{T} 3+\mathrm{CC}$ group compared with the ALD+T3 group ( $\mathrm{P}<0.05$; Fig. 5B). Conversely, the expression of pro-IL-1 $\beta$ was significantly increased in the ALD+T3 group compared with the ALD group and significantly increased in the ALD+T3+CC group compared with the ALD+T3 group 

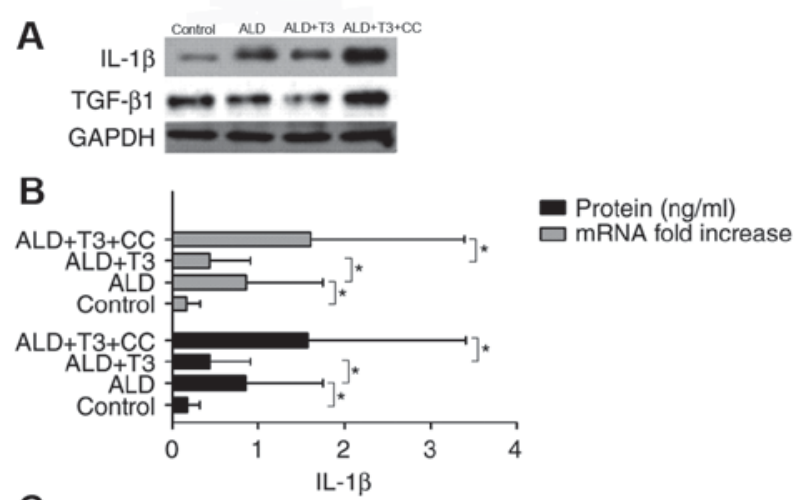

C

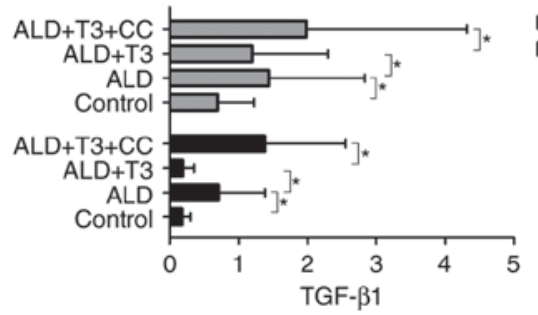

Figure 3. (A) Expression of IL- $1 \beta$ and TGF- $\beta 1$ protein in each group as measured by western blotting. Data was quantified and the protein and mRNA expression of (B) IL- $1 \beta$ and (C) TGF- $\beta 1$ in the liver tissues from each group was compared $(n=10)$. Data are expressed as the mean \pm standard deviation. ${ }^{*} \mathrm{P}<0.05$. IL, interleukin; TGF, transforming growth factor; ALD alcoholic liver disease; T3, triiodothyronine; CC, AMP-activated protein kinase inhibitor.

(both $\mathrm{P}<0.05$ ). Previous studies have demonstrated that NLRP3 activates pro-caspase- 1 into caspase- 1 , which can mediate hepatocyte apoptosis (23) and promote the transformation of pro-IL-1 $\beta$ into IL- $1 \beta^{8}$, which aggravates inflammatory injury in liver tissues. These results suggest that T3 alleviates liver injury in mice with alcoholic cirrhosis via negative regulation of the NLRP3 signaling pathway; however, this effect may be blocked by $\mathrm{CC}$.

\section{Discussion}

A total of 10 patients with liver cirrhosis were randomly selected and their ALT/AST levels were detected. Patients were treated with T3 $(0.1 \mathrm{mg} / \mathrm{kg} /$ day $)$ orally for 2 weeks. Further blood samples were taken and ALT/AST content prior to and following treatment was compared. It was revealed that ALT/AST content was significantly decreased in patients following T3 application compared with the baseline. Animal studies were performed to investigate the potential mechanisms that may be responsible for this.

It was observed that $\mathrm{T} 3$ significantly reduced serum transaminase and TBIL levels in ALD mice, which indicated that $\mathrm{T} 3$ effectively reduced the liver damage caused by ALD. However, the effect of $\mathrm{T} 3$ treatment was significantly blocked by $\mathrm{CC}$. In addition, morphological observations of liver tissues revealed that the number of hepatic lobules in the ALD+T3 group was significantly reduced compared with the ALD group, whereas they were significantly increased in the ALD+T3+CC group, which suggested that T3 alleviates the severity of liver fibrosis in ALD mice. These results indicate that T3 improved liver function and reduced the severity of liver cirrhosis in ALD mice, while this effect was blocked by CC. The possible mechanism responsible for the effect of T3 on ALD mice was then investigated.

Previous studies have demonstrated that chronic alcohol ingestion causes liver injury and increases the levels of ALT/AST and TBIL $(24,25)$. Animal experiments have also revealed that the increase in ALT/AST and TBIL may be associated with inflammation and apoptosis triggered by endoplasmic reticulum stress $(26,27)$. It was recently reported that T3 ameliorates ischemia-reperfusion injury by suppressing NLRP3 activation $(28,29)$. Therefore the authors hypothesized that the protective effects of T3 in ALD may involve inflammatory mediators and apoptosis factors.

IL-1 $\beta$ (4) and TGF- $\beta 1$ (20) are important inflammatory mediators that serve a role in the occurrence and development of ALD. In the present study, T3 significantly reduced the expression of IL- $1 \beta$ and TGF- $\beta 1$ mRNA and protein in the liver tissues from ALD mice, whereas its effect was blocked by $\mathrm{CC}$. These results suggest that the initial effect of T3 on ALD was to reduce the expression of IL- $1 \beta$ and TGF- $\beta 1$. It has been previously reported that NLRP3 is a key mediator of proinflammatory cytokine release (28). Previous studies have reported that caspase-1 promotes the transformation of pro-IL-1 $\beta$ into IL-1 $\beta$, which aggravates liver damage $(7,8)$. It has also been reported that NLRP3 upregulates the expression of caspase-1 (28). Based on these previous studies, the authors investigated whether the expression of IL-1 $\beta$ in ALD was associated with the regulation of the NLRP3-caspase-1 signaling pathway.

It was demonstrated that the expression of NLRP3, caspase- 1 and IL-1 $\beta$ in liver tissues was significantly decreased following T3 application in ALD mice, whereas the expression of pro-IL-1 $\beta$ was significantly increased. This suggests that T3 negatively regulates the NLRP3-caspase-1 signaling pathway, thereby significantly reducing the expression of IL-1 $\beta$. Previous study has reported that NLRP3 induces the activation of hepatic stellate cells, which is essential in the development of liver cirrhosis caused by ALD (30). The present study revealed that the mRNA and protein expression of $\alpha$-SMA in the ALD group was significantly increased compared with the control group. Following the application of T3, $\alpha$-SMA expression in the liver tissues of the mice was significantly decreased; however, the effect of T3 was blocked by CC. $\alpha$-SMA is an important marker of hepatic stellate cell activation (21), therefore these results suggest that T3 inhibits the production of TGF- $\beta 1$ and reduces the activation of hepatic stellate cells, thereby downregulating the expression of $\alpha$-SMA and alleviating the severity of liver cirrhosis.

Evidence suggests that the NLRP3 inflammasome serves a key role in the occurrence and development of tissue fibrosis (31). Gasse et al (32) demonstrated that the NLRP3 inflammasome existed in an activated state and was necessary during bleomycin-induced pulmonary fibrosis. In addition, previous studies have revealed that the NLRP3 inflammasome mediates the occurrence of fibrosis in systemic sclerosis ${ }^{24}$ and this can lead to cardiac infarction (33). A previous study using mice with a deleted NLRP3 gene revealed that the NLRP3 inflammasome induced liver fibrosis (33). Therefore, the NLRP3 downregulation observed in the present study may alleviate the severity of liver fibrosis in ALD. It has also been 


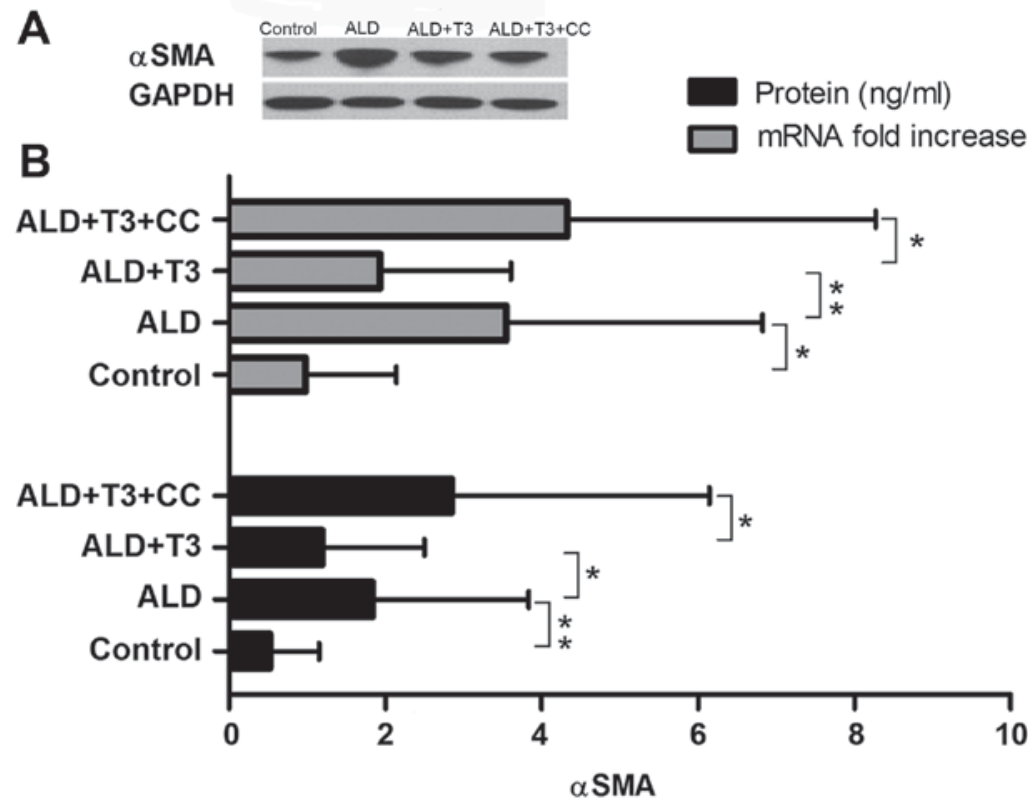

Figure 4. (A) Expression of $\alpha$-SMA protein in each group as determined by western blotting. (B) Data was quantified and the protein and mRNA expression of $\alpha$-SMA in liver tissues from each group was performed $(\mathrm{n}=10)$. Data are expressed as the mean \pm standard deviation. ${ }^{*} \mathrm{P}<0.05$ and ${ }^{* *} \mathrm{P}<0.01$. SMA, smooth muscle actin; ALD, alcoholic liver disease; T3, triiodothyronine; CC, AMP-activated protein kinase inhibitor.

A

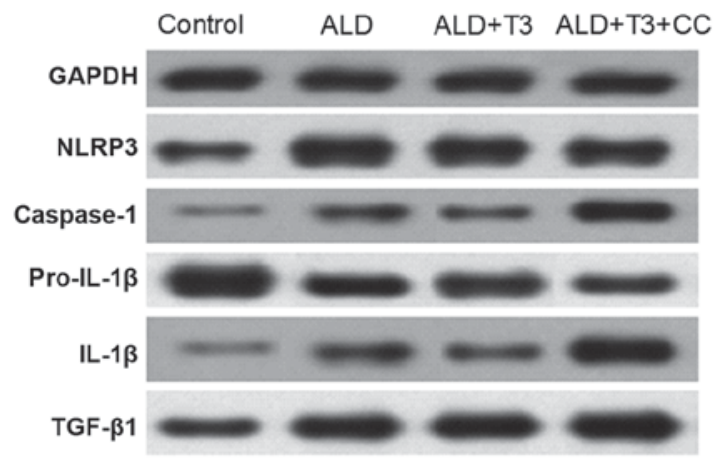

B

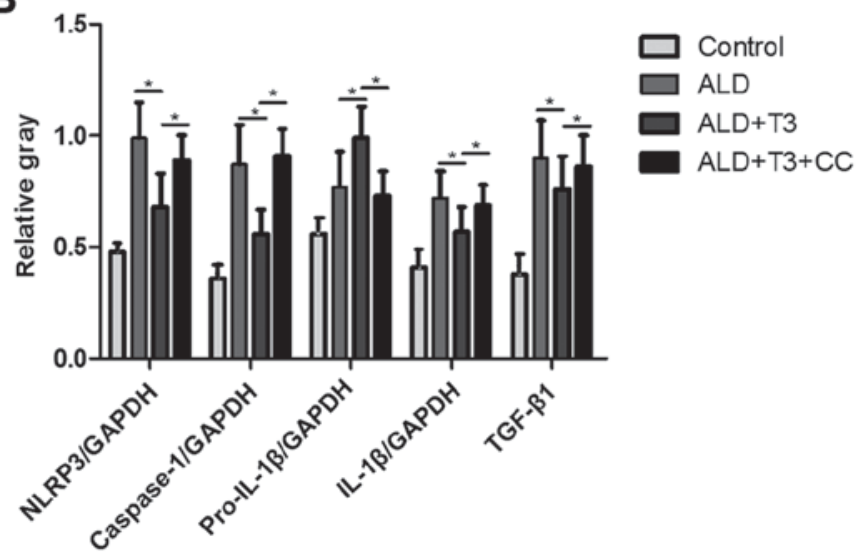

Figure 5. (A) Protein expression of NLRP3, caspase-1, pro-IL-1 $\beta$, IL-1 $\beta$ and TGF- $\beta 1$ as measured using western blotting. (B) Data was quantified using densitometry analysis $(n=10)$. Data are expressed as the mean \pm standard deviation. ${ }^{*} \mathrm{P}<0.05$. IL, interleukin; NLRP3, nucleotide-binding oligomerization domain, leucine rich repeat containing family, pyrin domain containing 3; TGF, transforming growth factor; ALD, alcoholic liver disease; T3, triiodothyronine; CC, AMP-activated protein kinase inhibitor.

reported that the NLRP3 inflammasome regulates the activation of hepatic stellate cells (34); studies have confirmed that activation of the NLRP3 inflammasome with monosodium urate crystals upregulates the expression of TGF- $\beta 1$ and collagen-1 (6). In the present study, the expression of NLRP3, TGF- $\beta 1$ and $\alpha$-SMA protein was significantly decreased following the application of T3, which may be blocked by CC. This indicates that T3 alleviates liver fibrosis in ALD, and that this is associated, at least in part, with negative regulation of the NLRP3-TGF- $\beta 1$ signaling pathway.

In conclusion, T3 alleviates the damage caused by ALD and its role may be associated with the fact that $\mathrm{T} 3$ negatively regulates the NLRP3 signaling pathway, thereby downregulating IL-1 $\beta$ and TGF- $\beta 1$ and reducing the activation of stellate cells. These findings provide a basis for a novel treatment option; in the future, hormone therapy may be used to relieve injury caused by alcoholic cirrhosis. However, there are several limitations in the present study, most notably the fact that only in vivo experiments were performed. These results should be supported by corresponding in vitro experiments. Further investigation is required to clarify the role of T3 and confirm its mechanism of action through in vitro experiments.

\section{Acknowledgements}

Not applicable.

\section{Funding}

No funding was received. 


\section{Availability of data and materials}

All data generated or analyzed during this study are included in this published article.

\section{Authors' contributions}

XD designed the study. HY collected the data. HY and XD performed the experiments. CL and QB analyzed and QL and $\mathrm{ZZ}$ interpreted the data. All authors read and approved the final version of the manuscript.

\section{Ethics approval and consent to participate}

This study was approved by the Medical Ethics Committee of Jining First People's Hospital (Jining, China). Patients or their guardians have provided written informed consent for publication.

\section{Patient consent for publication}

Not applicable.

\section{Competing interests}

The authors declare that they have no competing interests.

\section{References}

1. Federico A, Dallio M, Masarone M, Persico M and Loguercio C: The epidemiology of non-alcoholic fatty liver disease and its connection with cardiovascular disease: Role of endothelial dysfunction. Eur Rev Med Pharmacol Sci 20: 4731-4741, 2016.

2. Arsene D, Farooq O and Bataller R: New therapeutic targets in alcoholic hepatitis. Hepatol Int 10: 538-552, 2016.

3. Schwartz JM and Reinus JF: Prevalence and natural history of alcoholic liver disease. Clin Liver Dis 16: 659-666, 2012.

4. Petrasek J, Bala S, Csak T, Lippai D, Kodys K, Menashy V, Barrieau M, Min SY, Kurt-Jones EA and Szabo G: IL-1 receptor antagonist ameliorates inflammasome-dependent alcoholic steatohepatitis in mice. J Clin Invest 122: 3476-3489, 2012.

5. Kim EJ, Park SY, Baek SE, Jang MA, Lee WS, Bae SS, Kim K and Kim CD: HMGB1 increases IL-1 $\beta$ production in vascular smooth muscle cells via NLRP3 inflammasome. Front Physiol 9: 313,2018

6. Kim HY, Kim SJ and Lee SM: Activation of NLRP3 and AIM2 inflammasomes in Kupffer cells in hepatic ischemia/reperfusion. Febs J 282: 259-270, 2015.

7. Watanabe A, Sohail MA, Gomes DA, Hashmi A, Nagata J, Sutterwala FS, Mahmood S, Jhandier MN, Shi Y, Flavell RA and Mehal WZ: Inflammasome-mediated regulation of hepatic stellate cells. Am J Physiol Gastrointest Liver Physiol 296: G1248-G1257, 2009.

8. Esser N, Legrand-Poels S, Piette J, Scheen AJ and Paquot N: Inflammation as a link between obesity, metabolic syndrome and type 2 diabetes. Diabetes Res Clin Pract 105: 141-150, 2014.

9. Dinarello CA: Immunological and inflammatory functions of the interleukin-1 family. Annu Rev Immunol 27: 519-550, 2009.

10. Vargas R and Videla LA: Triiodothyronine suppresses ischemia-reperfusion-induced liver NLRP3 inflammasome activation: Role of AMP-activated protein kinase. Immunol Lett 184: 92-97, 2017.

11. Xiao J, Zhu Y, Liu Y, Tipoe GL, Xing F and So KF: Lycium barbarum polysaccharide attenuates alcoholic cellular injury through TXNIP-NLRP3 inflammasome pathway. Int J Biol Macromol 69: 73-78, 2014.

12. Cable EE, Finn PD, Stebbins JW, Hou J, Ito BR, van Poelje PD, Linemeyer DL and Erion MD: Reduction of hepatic steatosis in rats and mice after treatment with a liver-targeted receptor agonist. Hepatology 49: 407-417, 2009.
13. O'Shea RS, Dasarathy S and McCullough AJ: Alcoholic liver disease. AM J Gastroenterol 105: 14-32, 33, 2010

14. Rej R: Aspartate aminotransferase activity and isoenzyme proportions in human liver tissues. Clin Chem 24: 1971-1979, 1978.

15. Guesdon JL, Ternynck T and Avrameas S: The use of avidin-biotin interaction in immunoenzymatic techniques. J Histochem Cytochem 27: 1131-1139, 1979.

16. Kozutsumi Y, Segal M, Normington K, Gething MJ and Sambrook J: The presence of malfolded proteins in the endoplasmic reticulum signals the induction of glucose-regulated proteins. Nature 332: 462-464, 1988.

17. Tian X, Zhao C, Guo J, Xie S, Yin F, Huo X and Zhang X: Carvedilol attenuates the progression of hepatic fibrosis induced by bile duct ligation. Biomed Res Int 2017: 4612769, 2017.

18. Ke B, Shen XD, Zhang Y, Ji H, Gao F, Yue S, Kamo N, Zhai Y, Yamamoto M, Busuttil RW and Kupiec-Weglinski JW: KEAP1-NRF2 complex in ischemia-induced hepatocellular damage of mouse liver transplants. J Hepatol 59: 1200-1207, 2013.

19. Lee YA, Wallace MC and Friedman SL: Pathobiology of liver fibrosis: A translational success story. Gut 64: 830-841, 2015.

20. Wang H, Liu S, Wang Y, Chang B and Wang B: Nod-like receptor protein 3 inflammasome activation by Escherichia coli RNA induces transforming growth factor beta 1 secretion in hepatic stellate cells. Bosn J Basic Med Sci 16: 126-131, 2016.

21. Li J, Li J, Li S, He B, Mi Y, Cao H, Zhang C and Li L: Ameliorative effect of grape seed proanthocyanidin extract on thioacetamide-induced mouse hepatic fibrosis. Toxicol Lett 213: 353-360, 2012.

22. Jiao J, Friedman SL and Aloman C: Hepatic fibrosis. Curr Opin Gastroenterol 25: 223-229, 2009.

23. Dara L, Ji C and Kaplowitz N: The contribution of endoplasmic reticulum stress to liver diseases. Hepatology 53: 1752-1763, 2011.

24. Zhao S, Li N, Zhen Y, Ge M, Li Y, Yu B, He H and Shao RG: Protective effect of gastrodin on bile duct ligation-induced hepatic fibrosis in rats. Food Chem Toxicol 86: 202-207, 2015.

25. Wu JT, Yang GW, Qi CH, Zhou L, Hu JG and Wang MS: Anti-inflammatory activity of platycodin d on alcohol-induced fatty liver rats via tlr4-myd88-nf-kappab signal path. Afr J Tradit Complement Altern Med 13: 176-183, 2016.

26. Zhang W, Zhong W, Sun Q, Sun X and Zhou Z: Adipose-specific lipin1 overexpression in mice protects against alcohol-induced liver injury. Sci Rep 8: 408, 2018.

27. Chen X, Bian M, Zhang C, Kai J, Yao Z, Jin H, Lu C, Shao J, Chen A, Zhang $\mathrm{F}$ and Zheng S: Dihydroartemisinin inhibits ER stress-mediated mitochondrial pathway to attenuate hepatocyte lipoapoptosis via blocking the activation of the PI3K/Akt pathway. Biomed Pharmacother 97: 975-984, 2018.

28. Vargas R and Videla LA: Triiodothyronine suppresses ischemia-reperfusion-induced liver NLRP3 inflammasome activation: Role of AMP-activated protein kinase. Immunol Lett 184: 92-97, 2017.

29. Cannito S, Morello E, Bocca C, Foglia B, Benetti E, Novo E, Chiazza F, Rogazzo M, Fantozzi R, Povero D, et al: Microvesicles released from fat-laden cells promote activation of hepatocellular NLRP3 inflammasome: A pro-inflammatory link between lipotoxicity and non-alcoholic steatohepatitis. PLoS One 12: e172575, 2017.

30. Kawada N: Evolution of hepatic fibrosis research. Hepatol Res 41: 199-208, 2011.

31. Artlett CM: Inflammasomes in wound healing and fibrosis. J Pathol 229: 157-167, 2013.

32. Gasse P, Mary C, Guenon I, Noulin N, Charron S, Schnyder-Candrian S, Schnyder B, Akira S, Quesniaux VF, Lagente V, et al: IL-1R1/MyD88 signaling and the inflammasome are essential in pulmonary inflammation and fibrosis in mice. J Clin Invest 117: 3786-3799, 2007.

33. Shinde AV and Frangogiannis NG: Fibroblasts in myocardial infarction: A role in inflammation and repair. J Mol Cell Cardiol 70: 74-82, 2014.

34. Wree A, Eguchi A, McGeough MD, Pena CA, Johnson CD, Canbay A, Hoffman HM and Feldstein AE: NLRP3 inflammasome activation results in hepatocyte pyroptosis, liver inflammation, and fibrosis in mice. Hepatology 59: 898-910, 2014.

This work is licensed under a Creative Commons Attribution-NonCommercial-NoDerivatives 4.0 International (CC BY-NC-ND 4.0) License. 